\title{
Bivariate extreme value with application to PM10 concentration analysis
}

\begin{abstract}
This study is focus on a bivariate extreme of renormalized componentwise maxima with generalized extreme value distribution as a marginal function. The limiting joint distribution of several parametric models are presented. Maximum likelihood estimation is employed for parameter estimations and the best model is selected based on the Akaike Information Criterion. The weekly and monthly componentwise maxima series are extracted from the original observations of daily maxima PM10 data for two air quality monitoring stations located in Pasir Gudang and Johor Bahru. The 10 years data are considered for both stations from year 2001 to 2010. The asymmetric negative logistic model is found as the best fit bivariate extreme model for both weekly and monthly maxima componentwise series. However the dependence parameters show that the variables for weekly maxima series is more dependence to each other compared to the monthly maxima.
\end{abstract}

Keyword: Air quality; Akaike information criterion; Bivariate extreme; Maximum likelihood estimation; Parametric models 